\title{
PROSPECCIÓN CON GPR (GROUND PENETRATING RADAR) EN EL CEMENTERIO DE LAMISIÓN SALESIANA, RÍO GRANDE (TIERRA DEL FUEGO, ARGENTINA)
}

\author{
Alejandro Montes ${ }^{1}$, Mónica Salemme ${ }^{2}$, \\ Fernando Santiago ${ }^{3}$ y Ricardo Guichón ${ }^{4}$ \\ ${ }^{1}$ Depto Geología, F.C.N., U. N. Patagonia San Juan Bosco, Cdro. Rivadavia, E-mail: montes_alejandro_81@hotmail.com
2CADIC-CONICET, U.N. de Tierra del Fuego, B. Houssay 200, Ushuaia, E-mail: msalemme@cadic-conicet.gob.ar
${ }^{3}$ CADIC-CONICET, B. Houssay 200, Ushuaia, E-mail: ersant2@gmail.com
${ }^{4}$ Lab. de Ecología Evolutiva Humana, Fac. Cs. Soc., Univ. Nac. Del Centro, Quequén, E-mail: ricardoguichon@hotmail.com
}

Presentado el: 06/12/2011 - Aceptado 10/05/2012

\section{Resumen}

Se describen las tareas de prospección realizadas con GPR (Ground Penetrating Radar, o radar de penetración terrestre) en el Cementerio de La Misión Salesiana "La Candelaria", próximo a la localidad de Río Grande (Tierra del Fuego, Argentina). Este cementerio se encuentra ubicado sobre una planicie de cordones litorales del Holoceno, compuesta por depósitos de gravas y arenas con estratificación cruzada de bajo ángulo. Para las tareas de prospección se utilizó la antena de 400 MHz de frecuencia central del modelo SIR 3000 (GSSI 2004a). El objetivo consistió en identificar anomalías que permitieran corroborar la presencia, orientación y dimensiones de los enterramientos distribuidos en distintos sectores del cementerio, el cual contiene entierros de fines del siglo XIX y principios del siglo XX. En este trabajo se detallan principalmente los resultados obtenidos durante la segunda campaña realizada, en la cual se prospectó con GPR un sector donde, a partir de un sondeo exploratorio, se había registrado un enterramiento. Estos resultados servirán de base para tareas de prospección en otros sectores del cementerio contribuyendo con esta metodología a reducir el área a sondear con métodos arqueológicos tradicionales.

Palabras claves: GPR, Cementerio La Candelaria, cordones litorales, Tierra del Fuego.

\begin{abstract}
This paper describes the geophysical survey through GPR (Ground Penetrating Radar) in La Misión Salesiana cemetery, close to Río Grande city (Tierra del Fuego, Argentina). It is situated over the Holocene beach reach plain composed with low angle interstratifications of gravel and sand. A $400 \mathrm{MHz}$ antenna of the SIR 3000 model is the tool used for the survey. The main goal is to detect anomalies that allow corroborating the presence, orientation and dimension of the wood coffin found during a previous test pit, associated with burials of the late nineteenth and early twentieth centuries. This contribution represents a trial detailing the results obtained during the second season where the surveyed area was previously known from the presence of a burial. These results provide the basis for further exploration tasks in other sectors of the cemetery from which the use of this methodology will reduce the area to dig with traditional archaeological methods.
\end{abstract}

Keywords: GPR, Misión Salesiana Cemetery, littoral beach ridges, Tierra del Fuego 


\section{Introducción}

La antigua Misión Salesiana “Nuestra Señora de la Candelaria" ubicada en Río Grande (Tierra del Fuego, lat. $-53.724727^{\circ}$, long. $-67.798638^{\circ}$ ) funcionó como tal desde fines del siglo XIX, albergando tanto una población salesiana como pobladores originarios (Selk'nam). La misma contaba con un cementerio donde eran enterrados tanto indígenas como sacerdotes y monjas salesianos y, hasta mediados del XX, pobladores de la ciudad de Río Grande. Resulta, por tanto, un lugar relevante para fortalecer el conocimiento sobre los cambios epidemiológicos que se desarrollaron antes y durante el proceso de contacto interétnico en la región. Para iniciar estas tareas de investigación se contó con el permiso provincial de la autoridad de aplicación, el interés de las autoridades de la Misión a nivel local y se informó a representantes de la comunidad Selk'nam de Río Grande.

Casi conjuntamente con los trabajos sobre fuentes documentales (Casali et al. 2006, Casali y Guichón 2007, Casali et al. 2009, Casali 2011) en 2007 se iniciaron los primeros sondeos en el cementerio de la Misión Salesiana. Asimismo, en el marco de tareas de colaboración entre grupos de investigación del CONICET (Laboratorio de Geomorfología y Cuaternario, CADIC-CONICET y Laboratorio de Ecología Evolutiva Humana Quequen, FACSO-UNCPBA) se acordó evaluar las posibilidades de aplicar una técnica no invasiva en el mencionado cementerio con un equipo geofísico (Ground Penetrating Radar, GPR) para ubicar los enterratorios, especialmente los ataúdes.

El empleo del GPR ha experimentado un importante crecimiento en los últimos años. Se han obtenido interesantes resultados para la disciplina arqueológica, sobre todo en la detección de estructuras soterradas correspondientes a sitios históricos (Aguilera et al. 2006, Buscaglia 2001, Buscaglia et al. 2004, Conyers 2004, Domenico et al. 2004, Persson y Olofsson 2004, Vadis et al. 2005). En el norte de la Isla Grande de Tierra del Fuego y Patagonia Austral se puso a prueba esta tecnología utilizando antenas de $400 \mathrm{MHz}$ y $1,5 \mathrm{GHz}$ de frecuencia en sitios arqueológicos asociados a cazadores-recolectores (Salemme et al. 2006, Santiago et al. 2007, Santiago 2009, 2010) y se realizaron ensayos de laboratorio con sedimentos extraídos del sitio La Arcillosa 2 y materiales experimentales (Bujalesky et al. 2011).

El cementerio de la Misión Salesiana donde se realizó la prospección que aquí se describe, se ubica sobre una planicie de cordones (Figura 1) de grava y arena que representa un ambiente litoral regresivo asignado a la Formación San Sebastián definida por Codignotto (1969). Esta planicie de cordones del Holoceno posee un crecimiento por deriva hacia el sureste modificando a lo largo de $5 \mathrm{~km}$ la desembocadura del chorrillo La Misión (Bujalesky 2007). En este trabajo se presentan los resultados obtenidos en este contexto utilizando el GPR modelo SIR 3000 (Geophysical Survey Systems, Inc. 2004a) dotado de una antena de $400 \mathrm{MHz}$.

\section{Metodología}

La prospección geofísica con GPR en este sitio se realizó, como fue mencionado, utilizando una antena de $400 \mathrm{MHz}$ durante dos campañas de prospección realizadas en febrero de 2007 y enero de 2009. Dicha herramienta es un instrumento de prospección geofísica basado en la transmisión, reflexión y recepción de ondas electromagnéticas en un rango de frecuencias en el orden de los megahertz (MHz) a los gigahertz (GHz), a través de una antena emisora y una receptora. La primera emite energía electromagnética que se propaga a través del medio 


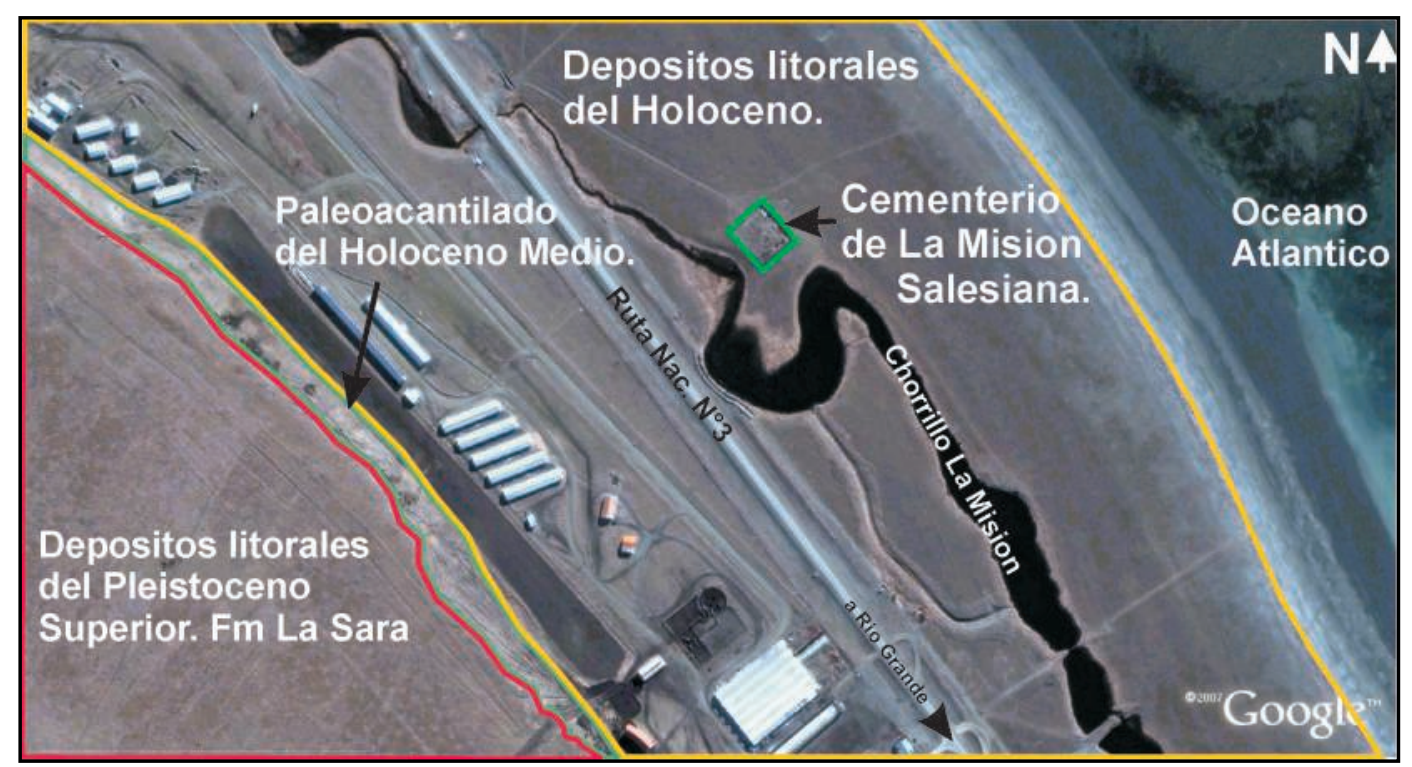

Figura 1. Imagen satelital de la zona en torno al Cementerio de La Misión Salesiana, Río Grande, Provincia de Tierra del Fuego.

a prospectar. La energía reflejada es detectada por la antena receptora y almacenada en la unidad de adquisición de datos. Dicha unidad posee una pantalla mediante la cual puede visualizarse el perfil que está siendo registrado. Este perfil cuenta con un eje horizontal en metros y un eje vertical en unidades de tiempo (TWTT, "Two-way Travel Time" o tiempo de ida y vuelta). Los principios de este método (colección de datos, procesamiento e interpretación) son descriptos en Davis y Annan (1989), Neal y Roberts (2001), Neal et al. (2002), Jol y Bristow (2003), Neal (2004), entre otros.

Las reflexiones se producen en subsuelo como resultado de las variaciones en las propiedades eléctricas de los materiales que lo componen. Cambios en la composición química de los sedimentos, en el tamaño de las partículas, en su forma, en su orientación, en su empaquetamiento, y en la presencia, distribución y contenido de los espacios porales (agua/aire), pueden generar cambios significativos en sus propiedades eléctricas (Neal et al. 2002, Jol y Bristow 2003, Neal 2004). Por lo tanto, las reflexiones pueden ser producidas por superficies en el subsuelo generadas por estructuras sedimentarias, contrastes litológicos, presencia del nivel freático o cuerpos muy reflectores o capaces de producir difracciones, como objetos, rasgos y/o estructuras de origen antrópico, lo que argumenta la hipótesis de trabajo planteada.

La cantidad de energía reflejada en las superficies mencionadas es proporcional al contraste entre las propiedades eléctricas de los materiales que separa. Cambios significativos en dichas propiedades quedarán representados por reflexiones intensas o absorciones fuertes. La principal propiedad que controla el comportamiento de la energía electromagnética en un medio es la permitividad dieléctrica relativa o constante dieléctrica $(\varepsilon)$.

Durante la campaña realizada en febrero de 2007 se diagramó una grilla de prospección en el sector suroeste del cementerio (Figura 2a-b). Se cubrieron $400 \mathrm{~m}^{2}$ trazando perfiles GPR de $40 \mathrm{~m}$ de largo, separando cada transecta por $50 \mathrm{~cm}$ de sur a norte y de 10 metros 
de largo con la misma separación de oeste a este. A su vez, fuera del predio del cementerio se realizó una grilla de prospección de $160 \mathrm{~m}^{2}$, con perfiles de $40 \mathrm{~m}$ de largo distanciados entre sí por $1 \mathrm{~m}$. Este primer ensayo se orientó a la puesta a punto de la técnica y a evaluar sus posibilidades. De esta manera se detectó una gran anomalía fuera del cementerio, sector que aún no fue excavado. Para optimizar el estudio dentro del predio se consideró necesario realizar una experiencia más acotada arealmente, ya que la gran cantidad de objetos presentes en subsuelo y en superficie (algunos de ellos metálicos) dificultan tanto la toma de datos como su interpretación. Por tal motivo en la campaña realizada en febrero de 2009 se delimitó la prospección a un sector donde la grilla cubrió una superficie de $20 \mathrm{~m}^{2}$ (Figura 3) trazando perfiles de $5 \mathrm{~m}$ de largo con un intervalo entre líneas de $50 \mathrm{~cm}$ y utilizando la misma antena y partiendo de un sondeo $(50 \times 50 \mathrm{~cm})$ previo, donde previamente se había

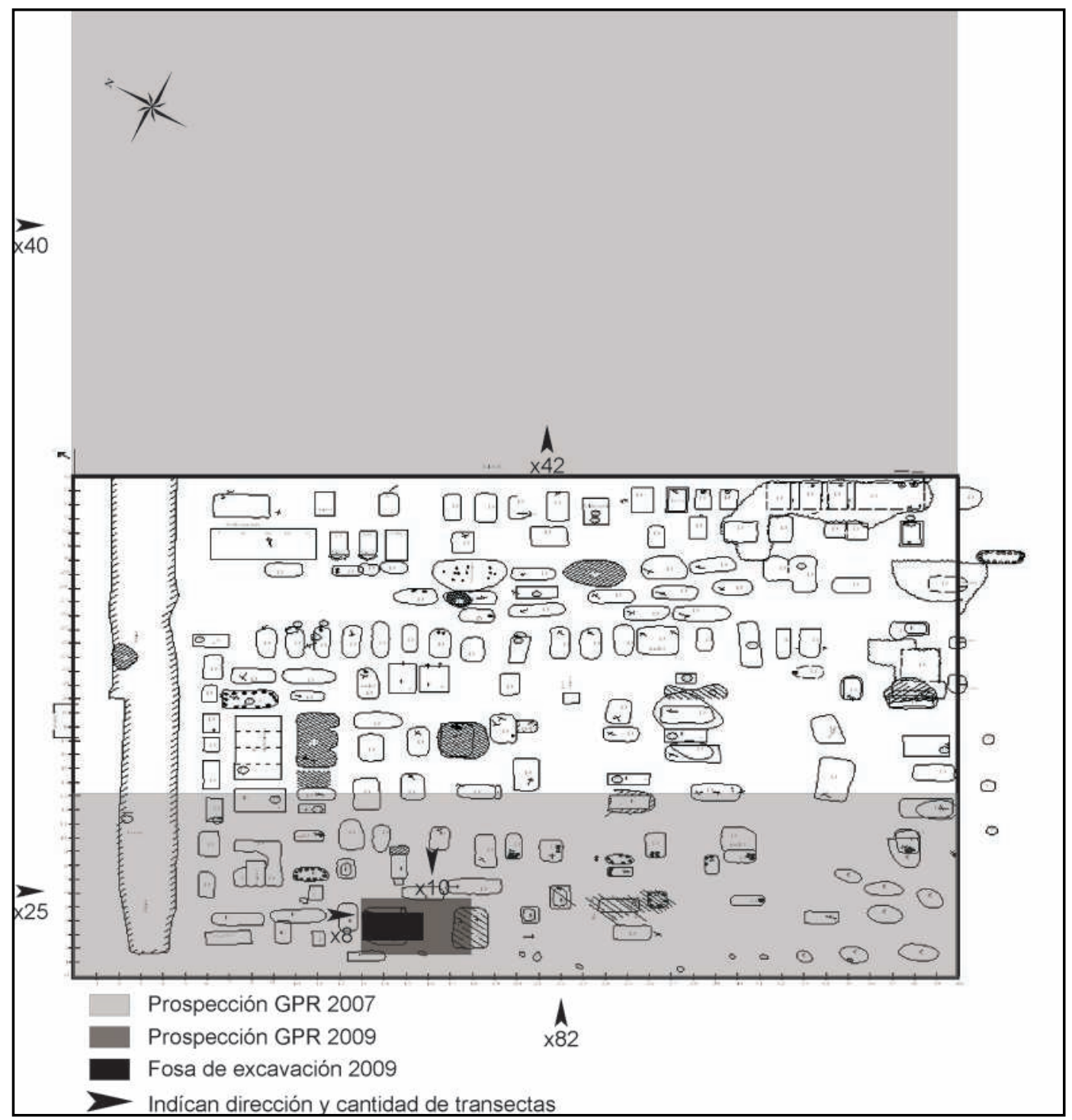

Figura 2. Plano del cementerio con la ubicación de las áreas prospectadas con GPR en 2007 (dentro y fuera del cementerio) y en 2009. Del sector pintado de negro procede un ataúd de madera con los restos humanos analizados. 
identificado el extremo de un ataúd a $100 \mathrm{~cm}$ de profundidad aproximadamente (Figura 2) El interés en esta primera etapa es explorar las posibilidades y limitaciones de esta técnica en este lugar en particular como estrategia para definir donde excavar nuevos enterratorios. Cabe señalar que de este sector del cementerio no se dispone de información de la ubicación exacta de los enterramientos.

Los perfiles GPR se realizaron con rumbo noreste-suroeste y noroeste-sureste, en forma perpendicular y paralela respectivamente a la orientación del ataúd, inferida a partir de las observaciones realizadas en el sondeo exploratorio. Luego de la prospección geofísica, el equipo de uno de nosotros (RG) excavó en este sector para analizar los restos humanos del enterramiento anteriormente detectado (Figura 2).

Se configuró la herramienta utilizando una amplitud de 35 ns, 40 escaneos por metro y 1024 muestreos por escaneo. Se fijó el apilamiento (stack) en 12 con filtros verticales en 100 $\mathrm{MHz}$ y $800 \mathrm{MHz}$ (filtro pasa altos y filtro pasa bajos, respectivamente) y se confeccionó la curva de ganancia con 5 puntos. El procesamiento de los registros se realizó mediante el software Radan 6.0 para Windows (GSSI 2004a). En ellos se corrigió la posición vertical, se ajustó la ganancia, realizándose distintas pruebas aplicando deconvolución y la transformada de Hilbert. Posteriormente se armaron distintos bloques 3D con registros GPR para hacer un análisis en 3 dimensiones del área prospectada. También se analizaron los registros obtenidos durante la campaña realizada en febrero de 2007 y las imágenes obtenidas a partir de su procesamiento. Se cuenta con un total de 207 registros GPR del cementerio y el sector contiguo ubicado al noreste.

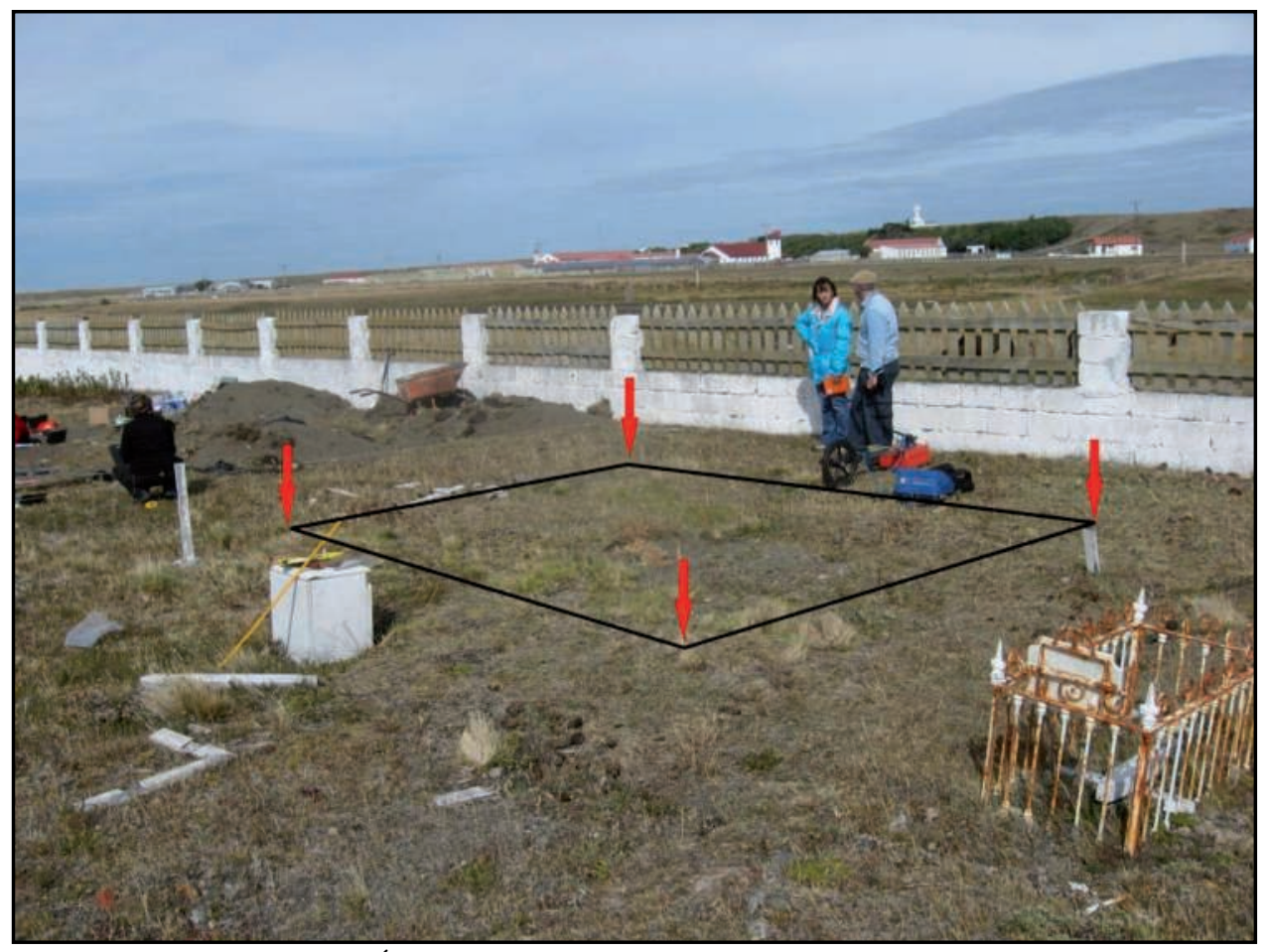

Figura 3. Área prospectada en campaña de febrero de 2009. 
Se utilizó un valor de 5,29 para la constante dieléctrica obtenido a partir de una medición de Punto Común Profundo (Common Mid Point, CMP) realizada en 2009 con dos antenas de $100 \mathrm{MHz}$ registrándose una velocidad de la onda en el medio de $0,130 \mathrm{~m} / \mathrm{ns}$. Esta medición se realizó a 11,2 Km al Norte, en la zona de la Estancia Las Violetas, sobre depósitos litorales equivalentes a los del área del cementerio en estudio, correspondientes a la Formación San Sebastián (Codignotto 1969). A su vez, el valor obtenido fue corroborado con la geometría de las reflexiones hiperbólicas detectadas en los distintos registros correspondientes en el cementerio y su entorno.

\section{Resultados}

A partir del análisis de los bloques 3D realizados con los registros obtenidos en la prospección de febrero de 2009 es posible identificar anomalías coincidentes con la posición de uno de los enterramientos del cementerio, reconocido a partir del sondeo antes mencionado. Las anomalías se observan en los cortes verticales de los bloques, tanto en dirección noreste-

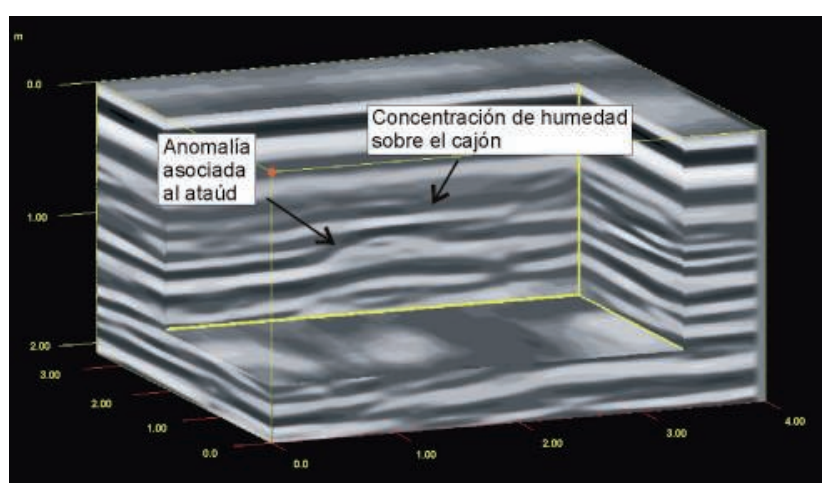

Figura 4. Bloque 3D construido con registros GPR. Se señala en el corte perpendicular a la orientación del ataúd una anomalía que coincide con su posición.

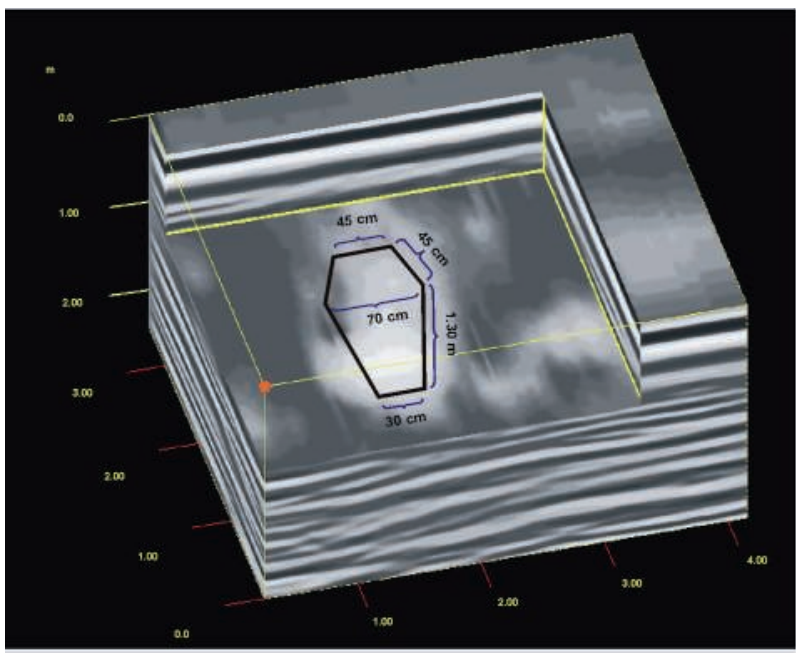

Figura 5. Superposición del esquema del ataúd desenterrado y un corte de tiempo en el bloque 3D donde se reconoce la anomalía asociada. sudoeste como noroeste-sureste. En el corte que se muestra en la figura 4 se observa la interrupción de reflectores a ambos lados de un reflector que adquiere mayor amplitud en el centro de la imagen. Dicho reflector es continuo, aunque cambia su morfología como puede verse en el sector señalado en dicha figura.

El ataúd de madera hallado estaba ubicado a una profundidad comprendida entre $1,2 \mathrm{~m}$ y $1,5 \mathrm{~m}$, por lo que coincide en forma aproximada con las anomalías descriptas (Figura 5). Su techo se encontraba colapsado y apoyado sobre un esqueleto humano completo y con vestimenta que finalmente fue recuperado en excavación semanas después de esta prospección geofísica.

Inmediatamente por encima de dichas anomalías se identifica otro reflector también de una amplitud considerable, la cual se va perdiendo lateralmente. Este reflector correspondería a un sector de mayor humedad en los sedimentos, asociado a la infiltración interferida por la presencia del ataúd.

Este sector con anomalías también se reconoce en los cortes de tiempo 
(Figura 5). La posición, profundidad y orientación del ataúd se asemejan a lo corroborado durante la excavación. A su vez, las dimensiones del ataúd se corresponden en forma aproximada con la distribución de la anomalía detectada.

Además de las anomalías mencionadas e identificadas a partir del análisis del bloque 3D confeccionado, mediante el estudio de los radargramas en forma individual es posible identificar hipérbolas generadas por la interrupción del frente de ondas electromagnéticas al

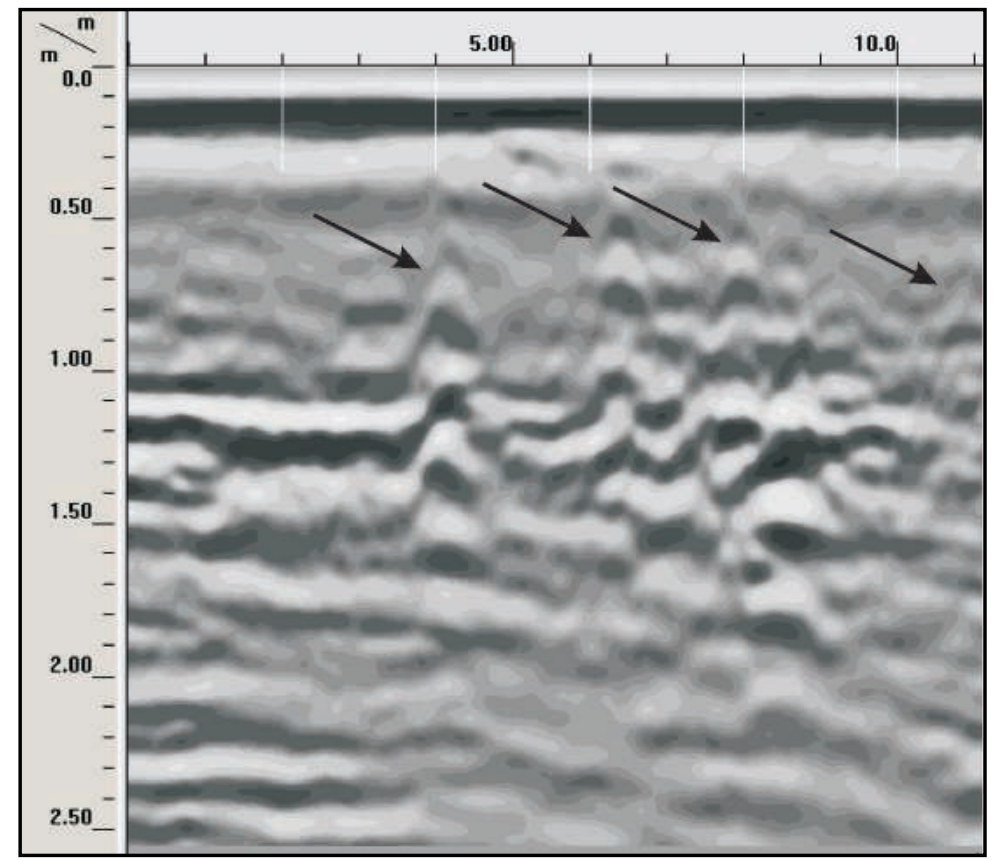

Figura 6. Registro orientado sudoeste-noreste donde se señalan hipérbolas de difracción asociadas a la intercepción de la onda radar con objetos soterrados. Por debajo de 1,40 $\mathrm{m}$ de profundidad se reconocen reflectores con suave inclinación al noreste, asociados a los depósitos litorales.

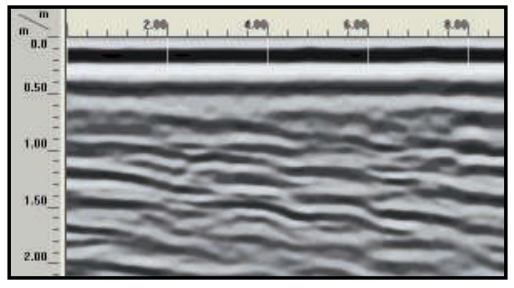

Figura 7. Reflectores con suave inclinación al noreste $\left(\approx 4,7^{\circ}\right)$ asociados a depósitos litorales correspondientes a un ambiente intermareal alto registrados fuera del predio del cementerio en un perfil GPR con orientación sudoeste-noreste. interceptar objetos ubicados en el subsuelo. Dichas hipérbolas se generan a profundidades entre los 0,50 y 1,4 m a lo largo de los registros obtenidos tanto, en la presente campaña de prospección como en los correspondientes a la realizada durante febrero de 2007 (Figura 6). Estas hipérbolas podrían corresponder tanto a objetos enterrados como a guijarros y bloques de origen litoral como los que se observan en la figura 8. En profundidades superiores a 1,40 m se reconocen reflectores asociados a los depósitos litorales del Holoceno sobre los cuales se organizó el cementerio.

Al tratarse de depósitos litorales regresivos de la costa Atlántica, alineados con la costa actual de rumbo noroeste-sureste, los reflectores en los radargramas 


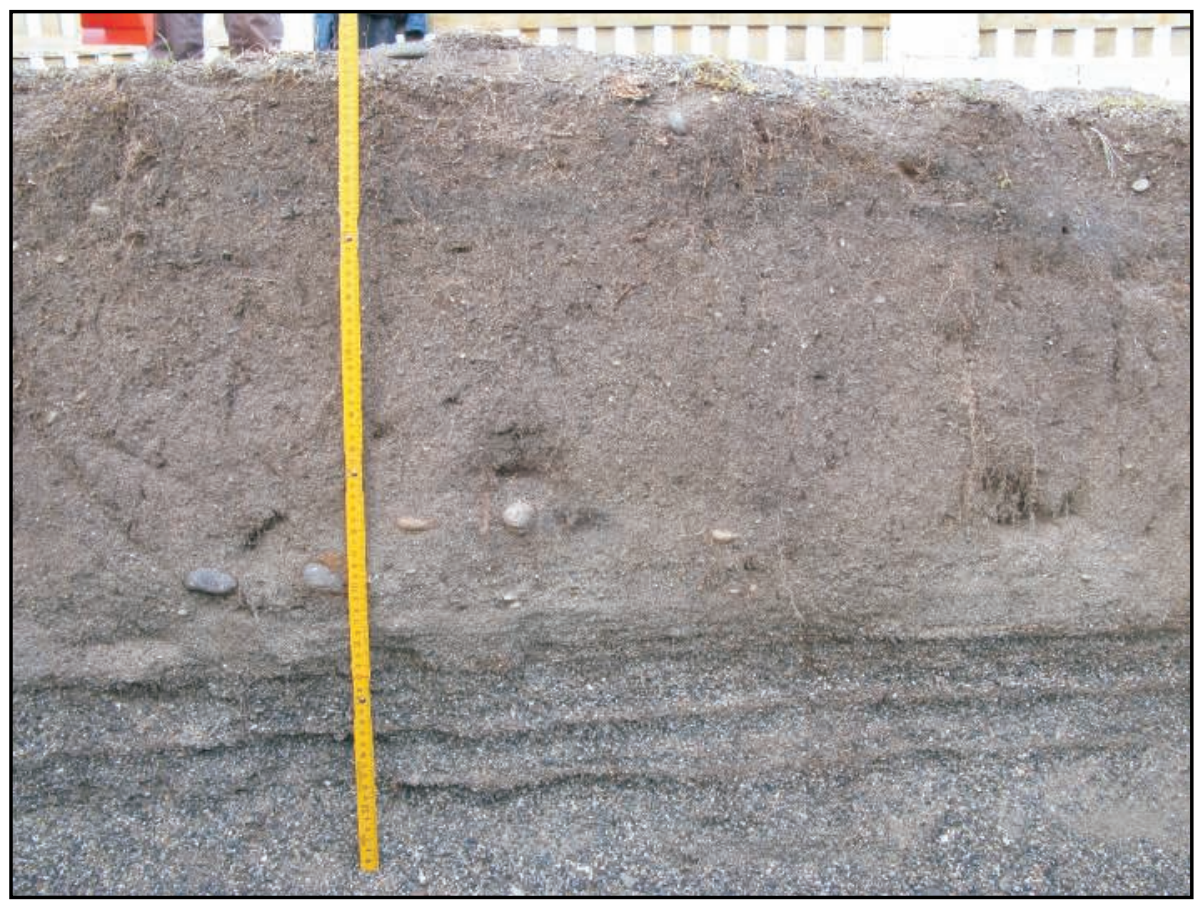

Figura 8. Grava y arena con estratificación cruzada de muy bajo ángulo cubierta por sedimentos é́licos y litorales removidos dentro del predio del cementerio.

presentan una suave inclinación hacia el noreste (de 0 a $4,8^{\circ}$ ) la cual puede constatarse en los registros orientados en sentido noreste-sudoeste (Figura 7). Los mismos representan superficies de estratificación asociadas a un ambiente intermareal alto y supramareal (Figura 8). En los registros realizados en sentido noroeste-sureste los reflectores son horizontales ya que son paralelos al rumbo de las estructuras sedimentarias y a los cordones litorales que conforman la planicie.

\section{Discusión}

La prospección con GPR, como método geofísico indirecto y no invasivo, permite la identificación de anomalías en subsuelo que pueden corresponderse, como en el caso en estudio, con restos humanos enterrados hace un siglo aproximadamente. Estas anomalías posibilitan la estimación, en forma aproximada, de las dimensiones, profundidad y orientación de los objetos ubicados en subsuelo, dependiendo siempre de las condiciones del medio. A su vez, también permite diferenciar zonas disturbadas, en este caso antrópicamente, de sectores donde se conservan inalteradas las estructuras sedimentarias.

La litología -predominantemente conformada por arenas y gravas- permite la obtención de registros GPR cuyos reflectores responden claramente a su arreglo sedimentario interno. El contraste entre las propiedades electromagnéticas de dichos sedimentos con las del ataúd de madera conteniendo restos humanos es lo suficientemente fuerte como para generar un óptimo contexto para la aplicación de este tipo de tecnologías.

A partir de la experiencia adquirida y en base a los resultados obtenidos se prevé realizar una nueva campaña de prospección geofísica con GPR. El objetivo principal será la pros- 
pección en la porción noreste del Cementerio ${ }^{1}$ para identificar potenciales sectores pasibles de excavación no visibles a partir de indicios superficiales. A su vez, la cantidad de objetos que aún permanecen tanto en superficie como en subsuelo y que afectan a la adquisición y procesamiento de los registros, sugieren avanzar sobre áreas de prospección específicas y de detalle, lo que optimiza las tareas de campo y de gabinete. Aplicando esta metodología se pretende minimizar el área afectada por las excavaciones reduciendo al máximo posible la alteración de este sitio histórico de alta sensibilidad cultural.

\section{Conclusiones}

1- Se corroboró la coincidencia de las anomalías detectadas en los registros GPR con uno de los ataúdes que contenía un cuerpo enterrado en el cementerio de La Misión Salesiana, tanto en los radargramas individuales como en los cortes verticales y horizontales de los bloques 3D confeccionados.

2- Mediante el análisis de estos bloques 3D puede reconocerse la orientación de los restos enterrados, presentando las anomalías asociadas una elongación coincidente con dichos restos.

3- Las dimensiones del cajón de entierro se corresponden en forma aproximada con las anomalías identificadas a partir del análisis de los bloques 3D.

4- Con el conocimiento de la respuesta del GPR en los depósitos circundantes al cementerio no afectados antrópicamente, y mediante la identificación de distintas anomalías (hipérbolas de difracción, variaciones en la morfología de los reflectores, presencia de reflectores difusos y reflectores de mayor amplitud que se pierden lateralmente) fue posible diferenciar las zonas disturbadas de los sectores vírgenes donde se conservan inalteradas las estructuras sedimentarias de los cordones de grava.

\section{Agradecimientos}

Las autoridades y personal de la Misión Salesiana facilitaron las instalaciones para alojamiento del grupo de trabajo. Al Padre Tico por su colaboración y apoyo. El Dr. G. Bujalesky (CADIC) leyó y comentó una versión preliminar de este trabajo. A Mariana Segura, Alejandra Ibáñez, Francisco Guichón, Martín H. Fugassa, y Jorge A. Suby por su colaboración en las campañas realizadas entre el 2007 y el 2009. El trabajo fue financiado a través de los proyectos PICT05-38096 (MS) y PICT 13889 y 01520 (RG).

\section{Notas}

${ }^{1}$ Cabe señalar que hasta el momento el equipo dirigido por el Dr. Guichón realizó 4 campañas $(2007,2008,2009$ y 2011) que permitieron obtener información sobre 21 individuos, la mayoría de los cuales se recuperaron dentro de cajones de madera (Guichón y Fugassa 2005, Guichón et al. 2006, Guichón et al. 2007, García Laborde et al. 2009, Laborde 2011).

\section{Bibliografía citada}

Aguilera D., A.Giaccardi, M.T.Carrara, N. De Grandis, D. Membrives y D. Codega 2006 Prospección geoeléctrica en manzana 38 del Parque Arqueológico "SFLV", Cayastá, Pcia. de Santa Fe. Estudios de Arqueología Histórica. Investigaciones argentinas pluridisciplinarias. $2^{\circ}$ Congreso de Arqueología Histórica Argentina: 259-267. Río Grande, Tierra del Fuego. 
Bujalesky, G.G.

2007 Coastal geomorphology and evolution of Tierra del Fuego (Southern Argentina). Geologica Acta 5 (4): 337-362. Barcelona, España.

Bujalesky G.G., A. Montes y F. Santiago

2011 Métodos de prospección geofísica en arqueología. Experimentación con radar de penetración terrestre (GPR) en concheros artificiales. Arqueología Iberoamericana (12): 3-14.

Buscaglia, S.

2001 Métodos geofísicos y propiedades del registro arqueológico. Arqueología no intrusiva en el sitio de Floridablanca (Pto. San Julián, Pcia. de Santa Cruz). Facultad de Filosofía y Letras. UBA. MS. Tesis de Licenciatura en Arqueología.

Buscaglia S., J.L. Lanata, X. Senatore, A. Osella, N.E. Lascano y M. De la Vega

2004 Prospecciones geofísicas en Floridablanca, San Julián, Pcia. de Santa Cruz. Ediciones Instituto Nacional de Antropología y Pensamiento Latinoamericano. Contra viento y marea. Arqueología de Patagonia (comp.por M.T. Civalero, G. Guraieb y P.M. Fernández), pp. 521536. Buenos Aires.

Casali, R.

2011 Contacto Interétnico en el Norte de Tierra del Fuego: la Misión Salesiana la Candelaria (Río Grande) y la salud de la población Selk'nam (1895-1931). Tesis doctoral, Facultad de Humanidades, UNMDP. Inédita.

Casali R., M.H. Fugassa y R.A. Guichón

2006 Aproximación epidemiológica al proceso de contacto interétnico en el norte de Tierra del Fuego. Magallania 34 (1): 141-155. Punta Arenas, Chile.

Casali R. y R.A. Guichón

2007 La misión salesiana La Candelaria y los cambios en la dieta de los Selk'nam. Octavas Jornadas Nacionales de Antropología Biológica. Salta, Argentina.

Casali R., M.H. Fugassa y R.A. Guichón

2009 Nuevos datos sobre la misión salesiana La Candelaria, Río Grande, Tierra del Fuego. Arqueología de la Patagonia. Una mirada desde el último confin (ed. por M. Salemme, F. Santiago, M. Álvarez, E. Piana, M. Vázquez y M.E. Mansur), pp. 577-591. Ushuaia.

Codignotto, J.O.

1969 Nota acerca de algunos aspectos geológicos de la costa patagónica comprendida entre Punta Loyola y el Cabo Vírgenes. Servicio de Hidrografía Naval. Armada Argentina, Buenos Aires.

Conyers, L. B.

2004 Ground-Penetrating Radar for Archaeology. Altamira Press, New York.

Cortez, M.

2003 Ground penetrating radar on Santa Cruz Island site scri-333. Geoscience Diversity Enhancement Project Symposium, Long Beach. 
Davis, J. y A. Annan

1989 Ground-penetrating radar for high-resolution mapping of soil and rock stratigraphy. Geophysical Prospecting 37: 531-551.

Domenico, C., M. D’Emilio, S. Di Salvia, V. Lapenna, M. Ragosta y E. Rizzo

2004 Magnetic mapping, ground penetrating radar surveys and magnetic susceptibility measurements for the study of the archaeological site of Serra di Vaglio (southern Italy). Journal of Archaeological Science 31: 633-643.

García Laborde, P., J.Suby, R. Guichón, N. Velázquez, L. Burry, P. Palacio, M.E. Segura y A. Ibáñez

2009 El cementerio de la antigua misión salesiana "La Candelaria” (Tierra del Fuego, Argentina). Campaña. Tercer Congreso de la Asociación de Paleopatología en Sudamérica. Necochea, Quequén, 14 al 16 de octubre de 2009.

García Laborde, P.

2011 El cementerio de la Antigua Misión Salesiana "Nuestra Señora de la Candelaria" (Rio Grande, Tierra del Fuego). Bioarqueología de contacto en el siglo XIX-XX. Tesis de licenciatura en Antropología, orientación Arqueología, Facultad de Ciencias Sociales, UNCPBA. Olavarría.

Guichón R. A. y M.H. Fugassa

2005 Informe sobre estudio del Cementerio La Candelaria. MS.

Guichón, R., J. Suby, R. Casali y M. Fugassa

2006 Health at the time of native-european contact in Southern Patagonia. Memorias do Instituto Oswaldo Cruz 101 (Suppl. II): 97-105. Río de Janeiro.

Guichón, R.A., M.H. Fugassa, J. Suby, R. Casali, A. Araujo y S. Mendonca De Souza

2007 The Cementery of "La Candelaria" Mission in Río Grande, Tierra del Fuego. II Congreso de la Asociación de Paleopatología en Sudamérica (PAMinSA II). Poster. Santiago de Chile.

Geophysical Survey Systems, Inc.

2004a RADAN for Windows. User's Manual. New Hampshire. North Salem. pp. 135.

Geophysical Survey Systems, Inc.

2004b TerraSIRch SIR System 3000: user's manuals. New Hampshire. North Salem. pp. 85

Jol, H. M. y C. Bristow

2003 GPR in sediments: advice on data collection, basic processing and interpretation. A good practice guide. Ground penetrating radar in sediments (ed. por C. Bristow y H. M. Jol), 211: 9-27. Geological Society of London. Special Publication, London.

Neal, A.

2004 Ground-penetrating radar and its use in Sedimentology: principles, problems and progress. Earth-Science Reviews 66: 261-330.

Neal, A. y C. Roberts

2001 Internal structure of a trough blowout, determined from migrated ground-penetrating radar profiles. Sedimentology 48: 791- 810. 
Neal, A., N. Pontee, K. Pye y J. Richards

2002 Internal structure of mixed-sand-and-gravel beach deposits revealed using groundpenetrating radar. Sedimentology 49: 789-804.

Pearsson, K. y B. Olofsson

2004 Inside a mound: Applied geophysics in archaeological prospecting at the Kings' Mounds, Gamla Uppsala, Sweden. Journal of Archaeological Science 31: 551-562.

Salemme, M., F. Santiago, L. Miotti y L. Magnin

2006 Prospección no invasiva en sitios a cielo abierto y en cuevas (Patagonia y Tierra del Fuego). III Congreso Argentino de Cuaternario y Geomorfología, Actas: 1-10, Córdoba.

Santiago, F.

2009 Dos sitios del norte de Tierra del Fuego "sondeados" con GPR: Río Chico 1 y La Arcillosa 2. Entre Pasados y Presentes II Estudios Contemporáneos en Ciencias Antropológicas (ed. por T. Bourlot, D. Bozzuto, C. Crespo, N. Kuperszmit y A. Hecht), pp. 171-183. Editorial Fundación Felix de Azara. Buenos Aires.

Santiago, F.

2010 La ocupación humana del norte de Tierra del Fuego durante el Holoceno medio y Tardío. Su vinculación con el paisaje. Tesis doctoral, Facultad de Cs. Sociales. Inédita. UNICEN. Olavarría.

Santiago, F., G. Bujalesky y M. Salemme 2007 Prospección arqueológica en la cuenca del río Chico. Tierra del Fuego, Argentina. Arqueología de Fuego-Patagonia. Levantando piedras, desenterrando huesos... y develando arcanos (ed. por F. Morello, A. Martinic, A. Prieto y G. Bahamonde), pp. 357-363. Ediciones CEQUA, Punta Arenas (Chile).

Vadis, A., N. Economou, Y. Ganiatsos, M. Manakou, G. Poulioudis, G. Sourlas, E. Vrontaki, A. Sarris, M. Guy, y T. Kalpaxis

2005 Integrated geophysical studies at ancient Itanos (Greece). Journal of

Archaeological Science. 32, 1023-1036. 